\section{Intermediate astronomy}

Astronomy: Principles and Practice. Pp. 341. Astronomy: Structure of the Universe. Pp. 283. By A. E. Roy and D. Clarke. (Adam Hilger: Bristol, UK, 1977.) Hardback $£ 10$; paperback $£ 7.50$ each volume.

Astronomy owes its educational value to a combination of separate factors: historical and literary associations, a wealth of comparatively easily understood applications of the scientific method, an increasingly significant relationship with the environment as revealed by modern space research, and its fundamental role in exploring what sort of Universe we live in. As Plato said, astronomy is good for the soul, and increasing recognition of this fact has led in recent years to the production of a large number of lively and beautifully illustrated books directed at 'liberal arts' students and bright youngsters, often making little or no use of formal mathematics; a few of these have been reviewed in Nature (266, 102, 3 March, 1977) by D. W. Hughes. For those with the mathematical equipment of a first-year honours student in physical sciences, an admirable compact survey of the whole field is provided by A. Unsöld's New Cosmos (of which an English version of the second edition is currently in press), whereas numerous other books deal with particular aspects in more detail.

The present two volumes by Roy and Clarke aim at an intermediate level. They are based on university and extramural lectures given by the authors at Glasgow over a number of years, and contain essentially the material of the first-year university course attended by beginning science students (who study a range of subjects at this stage) and a minority of arts students wishing to gain some knowledge of the workings of science and the scientific method. The first volume, beginning with nakedeye phenomena and a brief description of the observables, proceeds in classic Glaswegian tradition to give a thorough grounding in elementary spherical trigonometry, positional astronomy and celestial mechanics, including useful topical references to Megalithic observatories and space-probe orbits. A short introduction to atomic and radiation physics leads on to the second major section discussing optics, optical and radio telescopes, detectors and instruments, with a final chapter describing a number of practical projects, mostly concerned with positional astronomy but with some optics and spectroscopy as well, that involve very simple equipment. The second volume describes the objects and phenomena of astronomical research : members of the Solar System, tides and eclipses, stellar magnitudes, colours and spectroscopy, variable and binary stars, stellar atmospheres, interiors and evolution, stellar motions, nebulae, galactic structure, galaxies, cosmology, cosmogony and the question of extraterrestrial life.

Admirably illustrated and neatly packaged into 40 separate chapters with exercises, the two volumes are very successful in giving a clear exposition and in driving points home. The treatment and content, however, are not very well adapted to the needs of either honours students or liberal arts students, nor to those of bright youngsters. The mathematical approach is distinctly old-fashioned, with spherical triangles solved in remorseless detail (including logarithms of cosines written out in full), celestial mechanics without benefit of vector analysis, and trivial relationships involving stellar magnitudes spelled out at length.

The scope of the second volume is severely limited: to take just two ex-

\section{Viruses and cell surface changes}

Cell Surface Reviews. Volume 2 : Virus Infection and the Cell Surface. Edited by G. Poste and G. L. Nicolson. Pp. 342. (North-Holland: Amsterdam, New York and Oxford, 1977.) \$55.95; Dfl.137.

THE nature of surface changes induced, by interactions between viruses and cell membranes is currently a topic of considerable interest to many virologists and cellular immunologists. This follows the realisation that cellmediated immunity in virus diseases reflects surface monitoring of virally modified cells by re-circulating thymusderived lymphocytes ( $\mathrm{T}$ cells). Both viral and host components are apparently involved in $\mathrm{T}$ cell recognition. The evidence that has led to this concept is reviewed here by Blanden, Pang and Dunlop, and Burns and Allison. The area is, however, moving quickly and this volume is rather tardy, so some of the ideas expressed now have little currency.

The subject has not always been so fashionable. In fact, most of the virologists writing for this book are either unaware of, or have chosen to ignore, the trendy speculations of the cellular immunologists. This is somewhat of a relief. The result is a series of detailed reviews of established knowledge in areas that seem, in the main, to be relatively stable. The topics covered range through interactions of viruses with tissues (Smith, Weiner and John- amples, there is no mention of curves of growth or opacity in stellar atmospheres, and in the section on cosmology, General Relativity is merely mentioned as satisfying the classical observational tests without any accouni 5 the Principle of Equivalence or of any other idea connected with it, so that the notion of the curvature of space then comes right out of the blue. Thus the present book gives considerably less information on astrophysical topics than does the largely non-mathematical treatment of the entire field by G. O. Abell in Exploration of the Universe. The practical outlook, and the clarity and thoroughness of treatment (up to the chosen level) of those topics that have been selected for coverage, will, however, make this book a useful work of reference, especially for ambitious amateurs and for teachers of extramural courses.

Bernard Pagel

Bernard Pagel is Deputy Chief Scientific Officer at the Royal Greenwich Observatory, and Visiting Professor at the Astronomy Centre, University of Sussex, UK.

son), nucleated cells (Hosaka and Shimizu, Burns and Allison), erythrocytes (Bächi, Deas, and Howe) and model membrane systems (Tiffany). A thorough account of the structure and assembly of viral envelopes is provided by Rott and Klenk. Those considering the problem of immunological recognition of virally modified cells may benefit considerably from exposure to this information.

If I were to recommend, however, that a library acquire this very expensive volume, it is the article by Lindenmann that would induce me to do so. Lindenmann considers the various steps that led to general acceptance of the idea that a host-coded component, a carbohydrate moiety, is incorporated in budding influenza viruses. He uses this as a format to discuss both how science is done, and how credit is given (or denied) for what is done. The article is extremely readable, being written with some humour and considerable insight. This is recommended reading for students of virology, immunology and of the philosophy of science.

The book is well produced. Unfortunately, it has one major defect for those of us who read everything backwards. Individual articles are only identified at the beginning of each chapter. Printing of the authors names and/or a short title at the top of facing pages would make future volumes much more accessible for rapid reference.

P. C. Doherty

P. C. Doherty is Associate Professor at the Wistar Institute of Anatomy and Biology, Philadelphia, Pennsylvania. 